\title{
Research Article \\ Cluster Synchronization of Nonlinearly Coupled Complex Networks via Pinning Control
}

\author{
Jianwen Feng, ${ }^{1}$ Jingyi Wang, ${ }^{1}$ Chen $X_{u},{ }^{1}$ and Francis Austin ${ }^{2}$ \\ ${ }^{1}$ College of Mathematics and Computational Science, Shenzhen University, Shenzhen 518060, China \\ ${ }^{2}$ Department of Applied Mathematics, The Hong Kong Polytechnic University, Hong Kong
}

Correspondence should be addressed to Jianwen Feng, fengjw@szu.edu.cn

Received 18 June 2011; Revised 30 August 2011; Accepted 13 September 2011

Academic Editor: Recai Kilic

Copyright (C) 2011 Jianwen Feng et al. This is an open access article distributed under the Creative Commons Attribution License, which permits unrestricted use, distribution, and reproduction in any medium, provided the original work is properly cited.

We consider a method for driving general complex networks into prescribed cluster synchronization patterns by using pinning control. The coupling between the vertices of the network is nonlinear, and sufficient conditions are derived analytically for the attainment of cluster synchronization. We also propose an effective way of adapting the coupling strengths of complex networks. In addition, the critical combination of the control strength, the number of pinned nodes and coupling strength in each cluster are given by detailed analysis cluster synchronization of a special topological structure complex network. Our theoretical results are illustrated by numerical simulations.

\section{Introduction}

Complex networks synchronization is an important phenomenon in both mathematical and physical sciences because of its myriad applications to diverse problems such as communications security, seismology, parallel image processing as well as many others [1-7]. Loosely speaking, synchronization is the process in which two (or more) dynamical systems seek to adjust a certain prescribed property of their motion to a common behavior in the limit as time tends to infinity either by virtue of coupling or by forcing [8]. Some common synchronization patterns that have been widely studied are complete synchronization [9], lag synchronization [10], cluster synchronization [11], phase synchronization [12], and partial synchronization $[13,14]$.

Since Pecora and Carroll [15] found the chaos synchronization in 1990, synchronization has been widely studied because of its potential application in many different areas. Rulkov et al. [16] explored generalized synchronization of chaos in directionally coupled 
chaotic systems. (i.e., a response system is driven with the output of a driving system, but there is no feedback to the driver.) Hramov and Koronovskii [17] proposed an approach to the synchronization of chaotic oscillators based on the analysis of different time scales in the time series generated by the coupled chaotic oscillators and the quantitative measure of chaotic oscillator synchronous behavior.

In applications, many coupled oscillators are organized into subgroups called clusters, and cluster synchronization is the phenomenon in which all the nodes in one cluster are synchronized and those in different clusters are not. Cluster synchronization is an interesting phenomenon that has great application potentials, and many results have recently appeared that are of importance to our understanding of this problem. Ma et al. [18], for example, constructed a new coupling scheme that is capable of stabilizing every cluster synchronization pattern in a connected network with identical nodes by using cooperative and competitive coupling weights. Ma also derived a sufficient condition for the global stability of the synchronization patterns. Similarly, Wu et al. [19] showed how to construct the coupling matrix and modify the control strengths of a linearly coupled complex network with identical nodes by using pinning control. $\mathrm{Lu}$ et al. [20] investigated the cluster synchronization of dynamical networks with community structure and nonidentical nodes in the presence of time delays by applying the conventional feedback control schemes. Then, $\mathrm{Lu}$ et al. [21] carried on to study the cluster synchronization of general bi-directed networks with non-identical clusters and demonstrated a possible connection between the feasibility of cluster synchronization and the ratio of intra-to-inter cluster links of the network. Zhang et al. [22] found that different topologies of intercluster couplings might lead to different synchronizability, and different synchronous phases were revealed by varying the intercluster and intracluster coupling strengths.

Many cluster synchronization studies that have been conducted so far, however, have been confined to the smaller and less applicable class of complex networks that are linearly coupled [18-21] thus leaving the more important and naturally occurring nonlinearly coupled ones virtually uninvestigated. Nonlinearly coupled networks abound in nature (common examples are neural and metabolic networks in which the coupling configurations are oscillate continuously between two fixed states), and this paper attempts to fill this gap in our knowledge by investigating the nonlinearly coupled problem using pinning control. Pinning control is a very effective scheme for synchronizing chaos in complex networks by controlling only a small number of the system nodes [23], and it is a method that has been successfully applied to many spatially extended systems in the 1990s.

The main aim of this paper is to (nontrivially) extend the results of Wu et al. [19] to the more general problem of cluster synchronizing nonlinearly coupled complex networks by transforming the nonlinear coupling function into a linear. Our results are anticipated to be applicable to many cluster synchronization problems in various fields of science and technology.

This paper is organized as follows. In Section 2, we propose a model for nonlinearly coupled complex networks and state some necessary definitions, lemmas, and assumptions. In Section 3, we study the global cluster synchronization problem of networks by using pinning control and derive a sufficient condition for its attainment. Some adaptive feedback algorithms are also proposed for the coupling strengths of practical real-world networks. Numerical simulation results are presented in Section 4 . Section 5 concludes the paper. 


\section{Model and Preliminaries}

First, we give the mathematical definition of cluster synchronization.

Definition 2.1 (see $[18,19]$ ). Let $G_{1}=\left\{1,2, \ldots, k_{1}\right\}, G_{2}=\left\{k_{1}+1, k_{1}+2, \ldots, k_{1}+k_{2}\right\}, \ldots, G_{d}=$ $\left\{k_{1}+k_{2}+\cdots+k_{d-1}+1, \ldots, k_{1}+k_{2}+\cdots+k_{d}\right\}$ be a partition of the set $G=\{1,2, \ldots, m\}$ for $1<d<m, 1<k_{l}<m$, and $\sum_{l=1}^{d} k_{l}=m$, and, for every $i \in G$, let $\widetilde{i}$ be the counting index of the subset in which the number $i$ lies, that is, $i \in G_{i}$. A network with $m$ nodes is said to realize cluster synchronization with partition $\left\{G_{1}, G_{2}, \ldots, G_{d}\right\}$ if the state variables of the nodes satisfy $\lim _{t \rightarrow+\infty}\left\|x_{i}(t)-x_{j}(t)\right\|=0$ for $\tilde{i}=\tilde{j}$ and $\lim _{t \rightarrow+\infty}\left\|x_{i}(t)-x_{j}(t)\right\| \neq 0$ for $\tilde{i} \neq \tilde{j}$ for all initial values.

Next, we state the problem under consideration. A general nonlinearly coupled complex dynamical network with $m$ identical nodes can be described as

$$
\dot{x}_{i}(t)=f\left(x_{i}(t), t\right)+\sum_{j=1}^{m} a_{i j} \Gamma g\left(x_{j}(t)\right), \quad i=1,2, \ldots, m,
$$

where $x_{i}(t)=\left[x_{i}^{1}(t), x_{i}^{2}(t), \ldots, x_{i}^{n}(t)\right]^{T} \in R^{n}$ is the state vector of the $i$ th node, $f: R^{n} \times R^{+} \rightarrow R^{n}$ is a vector-valued function representing the activity of an individual subsystem, $g\left(x_{i}(t)\right)=$ $\left[h\left(x_{i}^{1}(t)\right), h\left(x_{i}^{2}(t)\right), \ldots, h\left(x_{i}^{n}(t)\right)\right]^{T}$ where $h: R \rightarrow R$ is a nonlinear function, $\Gamma=\left[\gamma_{i j}\right] \in R^{n \times n}$ is the inner coupling matrix, and $A=\left[a_{i j}\right] \in R^{m \times m}$ is the diffusive coupling matrix with $a_{i j}$ being the coupling weight along the edge from vertex $j$ to vertex $i$ and $a_{i i}=-\sum_{j=1, i \neq j}^{m} a_{i j}$ for $i, j=1,2, \ldots, m$.

When control is introduced, the nonlinearly coupling network (2.1) becomes

$$
\begin{gathered}
\dot{x}_{i}(t)=f\left(x_{i}(t), t\right)+\sum_{j=1}^{m} a_{i j} \Gamma g\left(x_{j}(t)\right)+u_{i}(t), \quad i \in J^{0}, \\
\dot{x}_{i}(t)=f\left(x_{i}(t), t\right)+\sum_{j=1}^{m} a_{i j} \Gamma g\left(x_{j}(t)\right), \quad i \notin J^{0},
\end{gathered}
$$

where $J^{0}$ is a subset of $\{1,2, \ldots, m\}$ denoting the controlled nodes set and $u_{i}(t)$ denotes the control on the node $i \in J^{0}$.

In this paper, we use the following approach to realize the given cluster synchronization pattern: (1) select $d$ special solutions $s_{1}(t), s_{2}(t), \ldots, s_{d}(t)$ of the homogenous system

$$
\dot{s}(t)=f(s(t), t)
$$

with distinct initial values such that $\lim _{t \rightarrow+\infty}\left\|s_{u}(t)-s_{v}(t)\right\| \neq 0$ for $u \neq v$. Then cluster synchronization is equivalent to the synchronization of the states $x_{i}(t)$ with the states $s_{i}(t)$, that is, $\lim _{t \rightarrow+\infty}\left\|x_{i}(t)-s_{\tilde{i}}(t)\right\|=0$ for $i=1,2, \ldots, m$. (2) Pinning controllers are added to some of the nodes in every cluster, without loss of generality, let the first $l_{u}\left(1 \leq l_{u} \leq k_{u}\right)$ nodes be selected to be pinned in every cluster; so let the controlled nodes set $J^{0}$ be $J=\bigcup_{u=1}^{d} J_{u}$, 
$J_{u}=\left\{b_{u-1}+1, b_{u-1}+2, \ldots, b_{u-1}+l_{u}\right\}, b_{0}=0$, and $b_{u}=\sum_{r=1}^{u} k_{r}$. We introduce nonlinear feedback pinning controllers

$$
u_{i}(t)=-\varepsilon_{\tilde{i}} \Gamma\left(g\left(x_{i}(t)\right)-g\left(s_{\tilde{i}}(t)\right)\right), \quad i \in J,
$$

where $\varepsilon_{\tilde{i}}>0$ with $i \in J$ are the control strengths. Then the following coupling system is considered:

$$
\begin{gathered}
\dot{x}_{i}(t)=f\left(x_{i}(t), t\right)+\sum_{j=1}^{m} a_{i j} \Gamma g\left(x_{j}(t)\right)-\varepsilon_{\tilde{i}} \Gamma\left(g\left(x_{i}(t)\right)-g\left(s_{\tilde{i}}(t)\right)\right), \quad i \in J, \\
\dot{x}_{i}(t)=f\left(x_{i}(t), t\right)+\sum_{j=1}^{m} a_{i j} \Gamma g\left(x_{j}(t)\right), \quad i \notin J .
\end{gathered}
$$

(3) Find sufficient conditions for the attainment of cluster synchronization for any initial value by pinning control.

The following are some definitions, lemmas, and notations which will be used throughout this paper.

Definition 2.2. If $A=\left[a_{i j}\right] \in R^{m \times m}$ is an irreducible matrix such that $a_{i j}=a_{j i} \geq 0$ for all $i \neq j$ and $\sum_{j=1}^{m} a_{i j}=0$ for all $i=1,2, \ldots, m$, then we say that $A \in \mathbf{A}_{\mathbf{1}}$.

Definition 2.3 (see $[18,19])$. Let

$$
A=\left[\begin{array}{cccc}
A_{11} & A_{12} & \cdots & A_{1 d} \\
A_{21} & A_{22} & \cdots & A_{2 d} \\
\vdots & \vdots & \ddots & \vdots \\
A_{d 1} & A_{d 2} & \cdots & A_{d d}
\end{array}\right]
$$

be an $m \times m$ symmetric matrix such that $A_{u v} \in R^{k_{u} \times k_{v}}, u, v=1,2, \ldots, d$. If each block $A_{u v}$ is a zero-row-sum matrix, then we say that $A \in \mathbf{M}_{\mathbf{1}}$. Furthermore, if $A_{u u} \in \mathbf{A}_{1}, u=1,2, \ldots, d$, then we say that $A \in \mathbf{M}_{2}$. It also follows from the symmetry of $A$ that $A_{u v}=A_{v u}^{T}$ and $A_{u v}, A_{v u}$ are zero-row-sum matrices and that $A_{u v}$ is a zero-column-sum matrix.

Definition 2.4 (see [24]). We denote $\mathbf{1}_{n}=[1,1, \ldots, 1]^{T} \in R^{n}, I_{n}=\operatorname{diag}\{1,1, \ldots, 1\} \in R^{n \times n}$, and define

$$
Q_{n}=I_{n}-\frac{1}{n} \mathbf{1}_{n} \cdot \mathbf{1}_{n}^{T} \in R^{n \times n}
$$

Obviously, $-Q_{n} \in \mathbf{A}_{\mathbf{1}}$. Moreover, it is easy to check that if $M \in R^{m \times n}$ is a zero-row-sum matrix, then $M Q_{n}=M$. So we have

$$
x^{T} M y=x^{T} M Q_{n} y \leq \frac{1}{2}\left(x^{T} M M^{T} x+y^{T} Q_{n} y\right) .
$$

The eigenvalues of $Q_{n}$ are 0 with multiplicity 1 and 1 with multiplicity $n-1$. 
Definition 2.5 (see $[25,26]$ ). Let $P=\operatorname{diag}\left\{p_{1}, p_{2}, \ldots, p_{n}\right\}$ and $\Delta=\operatorname{diag}\left\{\delta_{1}, \delta_{2}, \ldots, \delta_{n}\right\}$ be positive definite diagonal matrices. A function $f: R^{n} \times R^{+} \rightarrow R^{n}$ is said to belong to $\operatorname{QUAD}(P, \Delta)$, denoted by $f(x, t) \in \operatorname{QUAD}(P, \Delta)$ if $f$ satisfies the inequality

$$
(x-y)^{T} P((f(x, t)-f(y, t))-\Delta(x-y)) \leq-\eta(x-y)^{T}(x-y)
$$

for some $\eta>0, x, y \in R^{n}$, and $t>0$.

Definition 2.5 is known to be a typical property that is possessed by many of the benchmark chaotic systems such as the Lorenz system, the Chen system, the Lü system, and the unified chaotic system.

Definition 2.6 (see [27]). A function $h: R \rightarrow R$ is said to belong to $\mathrm{UNI}(\underline{h}, \bar{h})$, denoted by $h(\cdot) \in \mathrm{UNI}(\underline{h}, \bar{h})$, if $h$ satisfies

$$
\bar{h} \geq \frac{h(x)-h(y)}{x-y} \geq \underline{h}>0
$$

for every $x, y \in R$ for some constants $\bar{h}$ and $\underline{h}$.

Lemma 2.7 (see $[25,26]$ ). If $A=\left[a_{i j}\right] \in R^{m \times m}$ is such that $a_{i j}=a_{j i}$ and $a_{i i}=-\sum_{j=1, i \neq j}^{m} a_{i j}, i, j=$ $1,2, \ldots, m$, then

$$
u^{T} A v=\sum_{i=1}^{m} \sum_{j=1}^{m} u_{i} a_{i j} v_{j}=-\sum_{j>i} a_{i j}\left(u_{i}-u_{j}\right)\left(v_{i}-v_{j}\right)
$$

for all vectors $u=\left[u_{1}, u_{2}, \ldots, u_{m}\right]^{T}$ and $v=\left[v_{1}, v_{2}, \ldots, v_{m}\right]^{T}$.

Lemma 2.8 (see $[25,26]$ ). If $A=\left[a_{i j}\right] \in \mathbf{A}_{\mathbf{1}}$ and $\varepsilon>0$, then all the eigenvalues of the matrix

$$
\tilde{A}=\left[\begin{array}{cccc}
a_{11}-\varepsilon & a_{12} & \ldots & a_{1 m} \\
a_{21} & a_{22} & \ldots & a_{2 m} \\
\vdots & \vdots & \ddots & \vdots \\
a_{m 1} & a_{m 2} & \ldots & a_{m m}
\end{array}\right]
$$

are negative.

\section{Main Result}

In the section, we derive a sufficient condition for the attainment of cluster synchronization by considering a coupling matrix under pinning control. 
First, we prove the following theorem which follows directly from matrix theory and the Lyapunov function theorem.

Theorem 3.1. Suppose that the coupling matrix $A$ in system (2.5) satisfies $A \in \mathbf{M}_{2}$ and the inner coupling matrix $\Gamma=\operatorname{diag}\left\{\gamma_{1}, \gamma_{2}, \ldots, \gamma_{n}\right\}$ with $\gamma_{i}>0(i=1,2, \ldots, n)$. Let $\Delta=\operatorname{diag}\left\{\delta_{1}, \delta_{2}, \ldots, \delta_{n}\right\}$ and let $P=\operatorname{diag}\left\{p_{1}, p_{2}, \ldots, p_{n}\right\}$ be positive definite diagonal matrices such that $f(x, t) \in$ $\operatorname{QUAD}(P, \Delta) ; h(\cdot) \in \operatorname{UNI}(\underline{h}, \bar{h})$ with $\bar{h} \geq \underline{h}>0$. If

$$
\frac{\max _{k} \delta_{k} I_{k_{u}}}{\min _{k} \gamma_{k}}+\underline{h}\left(A_{u u}-\Xi_{u}\right)+\sum_{v=u+1}^{d} A_{u v} A_{u v}^{T}+(u-1) \bar{h}^{2} Q_{k_{u}} \leq 0, \quad u=1,2, \ldots, d,
$$

where $Q_{k_{u}} \in R^{k_{u} \times k_{u}}$ is defined as in Definition 2.4 for $u=1,2, \ldots, d, \Xi_{u}=\operatorname{diag}\left\{\varepsilon_{b_{u-1}+1}, \varepsilon_{b_{u-1}+2}\right.$, $\left.\ldots, \varepsilon_{b_{u}}\right\} \in R^{k_{u} \times k_{u}}$ with $\varepsilon_{b_{u-1}+i}=\varepsilon_{u}>0$ if $b_{u-1}+i \in J_{u}$, and $\varepsilon_{b_{u-1}+i}=0$ otherwise. Then, the solutions $x_{1}(t), x_{2}(t), \ldots, x_{m}(t)$ of system (2.5) satisfy $\lim _{t \rightarrow+\infty}\left\|x_{i}(t)-s_{\tilde{i}}(t)\right\|=0$ for $i=1,2, \ldots, m$, that is, the complex network (2.5) is a realized cluster synchronization with partition $\left\{G_{1}, G_{2}, \ldots, G_{d}\right\}$.

Proof. Let $e_{i}(t)=x_{i}(t)-s_{\tilde{i}}(t), i=1,2, \ldots, m$. Then, subtracting system (2.3) from system (2.5) yields the error system

$$
\begin{gathered}
\dot{e}_{i}(t)=f\left(x_{i}(t), t\right)-f\left(s_{i}(t), t\right)+\sum_{j=1}^{m} a_{i j} \Gamma g\left(x_{j}(t)\right)-\varepsilon_{i} \Gamma\left(g\left(x_{i}(t)\right)-g\left(s_{i}(t)\right)\right), \quad i \in J, \\
\dot{e}_{i}(t)=f\left(x_{i}(t), t\right)-f\left(s_{\tilde{i}}(t), t\right)+\sum_{j=1}^{m} a_{i j} \Gamma g\left(x_{j}(t)\right), \quad i \notin J .
\end{gathered}
$$

Since $A \in \mathbf{M}_{2}$, this implies that

$$
\sum_{j=1}^{m} a_{i j} \Gamma g\left(s_{\tilde{j}}(t)\right)=\Gamma \sum_{u=1}^{d} \sum_{j \in G_{u}} a_{i j} g\left(s_{u}(t)\right)=0
$$

For all $u, v=1,2, \ldots, d, A_{u v}$ is both a zero-row-sum and a zero-column-sum matrix; therefore,

$$
\begin{array}{rlrl}
\sum_{i \in G_{u}} \sum_{j \in G_{v}} a_{i j} x_{i}^{k}(t) h\left(x_{i}^{k}(t)\right) & =0, & & \sum_{i \in G_{u}} \sum_{j \in G_{v}} a_{i j} s_{\tilde{i}}^{k}(t) h\left(x_{i}^{k}(t)\right)=0, \\
\sum_{i \in G_{u}} \sum_{j \in G_{v}} a_{i j} s_{\tilde{j}}^{k}(t) h\left(x_{i}^{k}(t)\right)=0, & & \sum_{i \in G_{u}} \sum_{j \in G_{v}} a_{i j} x_{i}^{k}(t) h\left(s_{\tilde{i}}^{k}(t)\right)=0, \\
\sum_{i \in G_{u}} \sum_{j \in G_{v}} a_{i j} x_{i}^{k}(t) h\left(s_{\tilde{j}}^{k}(t)\right)=0, & & \sum_{i \in G_{u}} \sum_{j \in G_{v}} a_{i j} s_{\tilde{i}}^{k}(t) h\left(s_{\tilde{i}}^{k}(t)\right)=0 .
\end{array}
$$


Now let $\tilde{e}^{j}(t)=\left[e_{1}^{j}(t), e_{2}^{j}(t), \ldots, e_{m}^{j}(t)\right]^{T}$ and $\tilde{e}_{l}^{j}(t)=\left[e_{b_{l-1}+1}^{j}(t), e_{b_{l-1}+2}^{j}(t), \ldots, e_{b_{l}}^{j}(t)\right]^{T}$. Then, we obtain

$$
\begin{aligned}
\sum_{i=1}^{m} e_{i}(t)^{T} P \Delta e_{i}(t) & =\sum_{i=1}^{m} \sum_{k=1}^{n} p_{k} \delta_{k} e_{i}^{k}(t)^{2}=\sum_{k=1}^{n} p_{k} \delta_{k} \sum_{i=1}^{m} e_{i}^{k}(t)^{2} \\
& =\sum_{k=1}^{n} p_{k} \delta_{k} \tilde{e}^{k}(t)^{T} \tilde{e}^{k}(t)=\sum_{k=1}^{n} p_{k} \delta_{k} \sum_{l=1}^{d} \tilde{e}_{l}^{k}(t)^{T} \tilde{e}_{l}^{k}(t) .
\end{aligned}
$$

Next, define a Lyapunov function by

$$
V(t)=\frac{1}{2} \sum_{i=1}^{m} e_{i}(t)^{T} P e_{i}(t)
$$

and differentiate $V(t)$ along system (3.2) to get

$$
\begin{aligned}
\frac{d V(t)}{d t}= & \left\{\sum_{i=1}^{m} e_{i}(t)^{T} P\left[f\left(x_{i}(t), t\right)-f\left(s_{i}(t), t\right)-\Delta e_{i}(t)\right]+\sum_{i=1}^{m} e_{i}(t)^{T} P \Delta \mathrm{e}_{i}(t)\right\} \\
& +\sum_{i=1}^{m} e_{i}(t)^{T} P \sum_{j=1}^{m} a_{i j} \Gamma g\left(x_{j}(t)\right)-\sum_{i \in J} \varepsilon_{i} e_{i}(t)^{T} P \Gamma\left(g\left(x_{i}(t)\right)-g\left(s_{\tilde{i}}(t)\right)\right) \\
= & V_{1}(t)+V_{2}(t)+V_{3}(t) .
\end{aligned}
$$

Note that $f(x, t) \in \operatorname{QUAD}(P, \Delta)$, and from equality (3.5), we have

$$
\begin{aligned}
V_{1}(t) & =\sum_{i=1}^{m} e_{i}(t)^{T} P\left[f\left(x_{i}(t), t\right)-f\left(s_{i}(t), t\right)-\Delta e_{i}(t)\right]+\sum_{i=1}^{m} e_{i}(t)^{T} P \Delta e_{i}(t) \\
& \leq-\eta \sum_{i=1}^{m} e_{i}(t)^{T} e_{i}(t)+\sum_{k=1}^{n} p_{k} \delta_{k} \sum_{l=1}^{d} \tilde{e}_{l}^{k}(t)^{T} \tilde{e}_{l}^{k}(t) .
\end{aligned}
$$

For all $\tilde{x}^{j}(t)=\left[x_{1}^{j}(t), x_{2}^{j}(t), \ldots, x_{m}^{j}(t)\right]^{T}, \tilde{x}_{u}^{j}(t)=\left[x_{b_{u-1}+1}^{j}(t), x_{b_{u-1}+2}^{j}(t), \ldots, x_{b_{u}}^{j}(t)\right]^{T}, \tilde{h}\left(\tilde{x}_{u}^{j}(t)\right)=$ $\left[h\left(x_{b_{u-1}+1}^{j}(t)\right), h\left(x_{b_{u-1}+2}^{j}(t)\right), \ldots, h\left(x_{b_{u}}^{j}(t)\right)\right]^{T}, \tilde{s}_{u}^{j}(t)=\left[s_{u}^{j}(t), s_{u}^{j}(t), \ldots, s_{u}^{j}(t)\right]^{T} \in R^{k_{u}}, u=$ $1,2, \ldots, d$, and $\tilde{e}_{u}^{j}(t)=\tilde{x}_{u}^{j}(t)-\tilde{s}_{u}^{j}(t), j=1,2, \ldots, n$. It also follows that

$$
\begin{aligned}
V_{3}(t) & =-\sum_{i \in J} \varepsilon_{i} e_{i}(t)^{T} P \Gamma\left(g\left(x_{i}(t)\right)-g\left(s_{\tilde{i}}(t)\right)\right) \\
& \leq-\underline{h} \sum_{k=1}^{n} p_{k} \gamma_{k} \sum_{l=1}^{d} \tilde{e}_{l}^{k}(t)^{T} \Xi_{l} \tilde{e}_{l}^{k}(t),
\end{aligned}
$$


since $h(\cdot) \in \mathrm{UNI}(\bar{h}, \underline{h})$. Finally, we handle the most difficult $V_{2}(t)$, by using equality (3.3) and Lemma 2.7. More precisely, we get

$$
\begin{aligned}
V_{2}(t) & =\sum_{i=1}^{m} e_{i}(t)^{T} P \sum_{j=1}^{m} a_{i j} \Gamma g\left(x_{j}(t)\right) \\
& =\sum_{i=1}^{m} \sum_{j=1}^{m} a_{i j} e_{i}(t)^{T} P \Gamma\left(g\left(x_{j}(t)\right)-g\left(s_{\tilde{j}}(t)\right)\right) \\
& =\sum_{k=1}^{n} p_{k} \gamma_{k} \tilde{e}^{k}(t)^{T} A\left(g\left(\tilde{x}^{k}(t)\right)-g\left(\tilde{s}^{k}(t)\right)\right) \\
& =-\sum_{k=1}^{n} p_{k} \gamma_{k} \sum_{j>i} a_{i j}\left(e_{i}^{k}(t)-e_{j}^{k}(t)\right)\left(\left(h\left(x_{i}^{k}(t)\right)-h\left(s_{\tilde{i}}^{k}(t)\right)\right)-\left(h\left(x_{j}^{k}(t)\right)-h\left(s_{\tilde{j}}^{k}(t)\right)\right)\right) .
\end{aligned}
$$

Upon using the equalities (3.4), Lemma 2.7, and the fact that $h(\cdot) \in \mathrm{UNI}(\bar{h}, \underline{h})$, we obtain

$$
\begin{aligned}
V_{2}(t)=-\sum_{k=1}^{n} p_{k} \gamma_{k}\left\{\sum_{u=1}^{d} \sum_{i=b_{u-1}+1}^{b_{u}-1} \sum_{j=i+1}^{b_{u}} a_{i j}\left(x_{i}^{k}(t)-x_{j}^{k}(t)\right)\left(h\left(x_{i}^{k}(t)\right)-h\left(x_{j}^{k}(t)\right)\right)\right. \\
+\sum_{u=1}^{d-1} \sum_{v=u+1}^{d} \sum_{i=b_{u-1}+1}^{b_{u}} \sum_{j=b_{v-1}+1}^{b_{v}} a_{i j}\left(\left(x_{i}^{k}(t)-s_{\tilde{i}}^{k}(t)\right)-\left(x_{j}^{k}(t)-s_{\tilde{j}}^{k}(t)\right)\right) \\
\left.\quad \times\left(\left(h\left(x_{i}^{k}(t)\right)-h\left(s_{\tilde{i}}^{k}(t)\right)\right)-\left(h\left(x_{j}^{k}(t)\right)-h\left(s_{\tilde{j}}^{k}(t)\right)\right)\right)\right\} \\
\leq \sum_{k=1}^{n} p_{k} \gamma_{k}\left\{\sum_{u=1}^{d} \sum_{i=b_{u-1}+1}^{b_{u}-1} \sum_{j=i+1}^{b_{u}}-\underline{h} a_{i j}\left(x_{i}^{k}(t)-x_{j}^{k}(t)\right)^{2}\right. \\
\left.+\sum_{u=1}^{d-1} \sum_{v=u+1}^{d} \sum_{i=b_{u-1}+1}^{b_{u}} \sum_{j=b_{v-1}+1}^{b_{v}} 2 a_{i j} x_{i}^{k}(t) h\left(x_{j}^{k}(t)\right)\right\} \\
=\sum_{k=1}^{n} p_{k} \gamma_{k}\left\{\sum_{u=1}^{d} \underline{h} \tilde{x}_{u}^{k}(t)^{T} A_{u u} \tilde{x}_{u}^{k}(t)+\sum_{u=1}^{d-1} \sum_{v=u+1}^{d} 2 \tilde{x}_{u}^{k}(t)^{T} A_{u v} \tilde{h}\left(\tilde{x}_{v}^{k}(t)\right)\right\} \\
=\sum_{k=1}^{n} p_{k} \gamma_{k}\left\{\sum_{u=1}^{p} \underline{h} \tilde{e}_{u}^{k}(t)^{T} A_{u u} \tilde{e}_{u}^{k}(t)+\sum_{u=1}^{d-1} \sum_{v=u+1}^{d} 2 \tilde{e}_{u}^{k}(t)^{T} A_{u v} \widetilde{h}\left(\tilde{x}_{v}^{k}(t)\right)\right\},
\end{aligned}
$$


where

$$
\begin{aligned}
\sum_{u=1}^{d-1} \sum_{v=u+1}^{d} 2 \tilde{e}_{u}^{k}(t)^{T} A_{u v} \tilde{h}\left(\tilde{x}_{v}^{k}(t)\right) & \leq \sum_{u=1}^{d-1} \sum_{v=u+1}^{d}\left\{\tilde{e}_{u}^{k}(t)^{T} A_{u v} A_{u v}^{T} \tilde{e}_{u}^{k}(t)+\bar{h}^{2} \tilde{e}_{v}^{k}(t)^{T} Q_{k_{v}} \tilde{e}_{v}^{k}(t)\right\} \\
& \leq \sum_{u=1}^{d-1} \sum_{v=u+1}^{d} \tilde{e}_{u}^{k}(t)^{T} A_{u v} A_{u v}^{T} \tilde{e}_{u}^{k}(t)+\sum_{u=1}^{d-1} \sum_{v=u+1}^{d} \bar{h}^{2} \tilde{e}_{v}^{k}(t)^{T} Q_{k_{v}} \tilde{e}_{v}^{k}(t) \\
& =\sum_{u=1}^{d-1} \sum_{v=u+1}^{d} \tilde{e}_{u}^{k}(t)^{T} A_{u v} A_{u v}^{T} \tilde{e}_{u}^{k}(t)+\sum_{u=2}^{d} \sum_{v=1}^{u-1} \bar{h}^{2} \tilde{e}_{u}^{k}(t)^{T} Q_{k_{u}} \tilde{e}_{u}^{k}(t),
\end{aligned}
$$

for we have

$$
\begin{aligned}
2 \tilde{e}_{u}^{k}(t)^{T} A_{u v} \tilde{h}\left(\tilde{x}_{v}^{k}(t)\right) & =2 \tilde{e}_{u}^{k}(t)^{T} A_{u v} Q_{k_{v}} \tilde{h}\left(\tilde{x}_{v}^{k}(t)\right) \\
& \leq \tilde{e}_{u}^{k}(t)^{T} A_{u v} A_{u v}^{T} \tilde{e}_{u}^{k}(t)+\tilde{h}\left(\tilde{x}_{v}^{k}(t)\right)^{T} Q_{k_{v}} \tilde{h}\left(\tilde{x}_{v}^{k}(t)\right) \\
& \leq \tilde{e}_{u}^{k}(t)^{T} A_{u v} A_{u v}^{T} \tilde{e}_{u}^{k}(t)+\bar{h}^{2} \tilde{e}_{v}^{k}(t)^{T} Q_{k_{v}} \tilde{e}_{v}^{k}(t)
\end{aligned}
$$

(from Lemma 2.7 and inequality (2.8)).

Combining inequalities (3.11) and (3.12), we get

$$
V_{2}(t) \leq \sum_{k=1}^{n} p_{k} \gamma_{k} \sum_{u=1}^{d} \tilde{e}_{u}^{k}(t)^{T}\left\{\underline{h} A_{u u}+\sum_{v=u+1}^{d} A_{u v} A_{u v}^{T}+(u-1) \bar{h}^{2} Q_{k_{u}}\right\} \tilde{e}_{u}^{k}(t)
$$

and (upon substituting inequalities (3.8), (3.9), and (3.14) into equality (3.7)), we have

$$
\begin{aligned}
\frac{d V(t)}{d t} \leq & -\eta \sum_{i=1}^{m} e_{i}(t)^{T} e_{i}(t)+\sum_{k=1}^{n} p_{k} \delta_{k} \sum_{u=1}^{d} \tilde{e}_{u}^{k}(t)^{T} \tilde{e}_{u}^{k}(t) \\
& +\sum_{k=1}^{n} p_{k} \gamma_{k} \sum_{u=1}^{d}\left\{\underline{h} \tilde{e}_{u}^{k}(t)^{T} A_{u u} \tilde{e}_{u}^{k}(t)+\sum_{v=u+1}^{d} \tilde{e}_{u}^{k}(t)^{T} A_{u v} A_{u v}^{T} \tilde{e}_{u}^{k}(t)+(u-1) \bar{h}_{k}^{2} \tilde{e}_{u}^{k}(t)^{T} Q_{k_{u}} \tilde{e}_{u}^{k}(t)\right\} \\
& -\sum_{k=1}^{n} p_{k} \gamma_{k} \sum_{u=1}^{d} \tilde{e}_{u}^{k}(t)^{T} \Xi_{u} \tilde{e}_{u}^{k}(t) \\
= & -\eta \sum_{i=1}^{m} e_{i}(t)^{T} e_{i}(t) \\
& +\sum_{k=1}^{n} p_{k} \sum_{u=1}^{d} \tilde{e}_{u}^{k}(t)^{T}\left\{\delta_{k} I_{k_{u}}+\underline{h} \gamma_{k} A_{u u}-\underline{h} \gamma_{k} \Xi_{u}+\sum_{v=u-1}^{d} \gamma_{k} A_{u v} A_{u v}^{T}+(u-1) \bar{h}_{k}^{2} \gamma_{k} Q_{k_{u}}\right\} \tilde{e}_{u}^{k}(t) .
\end{aligned}
$$


Hence, by combining inequality (3.15) and condition (3.1), it gives

$$
\frac{d V(t)}{d t} \leq-\eta \sum_{i=1}^{m} e_{i}(t)^{T} e_{i}(t) \leq-\frac{2 \eta}{\max _{i} p_{i}} V(t)
$$

and then the solution $x_{1}(t), x_{2}(t), \ldots, x_{m}(t)$ of system (2.5) satisfies $\lim _{t \rightarrow \infty} \sum_{i=1}^{m} \| x_{i}(t)-$ $s_{\tilde{i}}(t) \|=0$ for all initial values.

Remark 3.2. It follows from Theorem 3.1 that every complex network with a coupling matrix satisfying inequality (3.1) can be cluster synchronized by pinning control. However, we should construct a coupling matrix $A \in \mathbf{M}_{2}$ and select suitable control strengths $\varepsilon_{1}, \varepsilon_{2}, \ldots, \varepsilon_{d}>0$, in order to make inequality (3.1) holds. It inspires that we can control some networks to achieve cluster synchronization by constructing a coupling matrix. For $i \neq j$, if $a_{i j} \neq 0$ with $i, j \in G_{u}$, then the coupling between oscillators $i$ and $j$ is called intercluster coupling, which can be regarded as a mechanism to synchronize $i$ and $j$. Since $A_{u u}-\Xi_{u}<$ 0 (from Lemma 2.8), the intercluster coupling strength should always be chosen to be controlled, and the following coupling matrix is constructed by adding partially connected (intercluster) coupling strengths

$$
\left[\begin{array}{cccc}
c A_{11} & A_{12} & \cdots & A_{1 d} \\
A_{21} & c A_{22} & \cdots & A_{2 d} \\
\vdots & \vdots & \ddots & \vdots \\
A_{d 1} & A_{d 2} & \cdots & c A_{d d}
\end{array}\right]
$$

for some positive constant $c[19,20]$. The control strengths are then $c \varepsilon_{l}, l=1,2, \ldots, d$. Furthermore, because it is usual for the theoretical value of $c$ to be much larger than its true value in applications (we shall show this in Section 4), it is important that $c$ be made as small as practically possible.

To make Theorem 3.1 more applicative, we give the following corollaries.

Corollary 3.3. Suppose $f(x, t) \in \operatorname{QUAD}(P, \Delta), h(\cdot) \in \operatorname{UNI}(\underline{h}, \bar{h}), \Gamma=\operatorname{diag}\left\{\gamma_{1}, \gamma_{2}, \ldots, \gamma_{n}\right\}$ is positive definite, (3.17) is the coupling matrix, and $Q_{k_{u}} \in R^{k_{u} \times k_{u}}$ is defined as in Definition 2.4; if the coupling strength satisfies

$$
c \geq \max _{1 \leq u \leq d} c_{u}
$$

where

$$
c_{u}=\max _{k}-\frac{\delta_{k}+\gamma_{k} \lambda_{\max }\left(\sum_{v=u-1}^{d} A_{u v} A_{u v}^{T}+\bar{h}^{2}(u-1) Q_{k_{u}}\right)}{\underline{h} \gamma_{k} \lambda_{\max }\left(A_{u u}-\Xi_{u}\right)},
$$

then system (2.5) can be cluster synchronized. 
When $\underline{h}=\bar{h}=1, h(x)=x$ is linear function; then system (2.5) become linearly coupling system; we have the following.

Corollary 3.4. Suppose $f(x, t) \in \operatorname{QUAD}(P, \Delta), h(\cdot) \in \operatorname{UNI}(1,1), \Gamma=I_{n}, Q_{k_{u}} \in R^{k_{u} \times k_{u}}$ is defined as in Definition 2.4, and (3.17) is the coupling matrix; if the coupling strength satisfies

$$
c \geq \max _{1 \leq u \leq d} c_{u}
$$

where

$$
c_{u}=-\frac{\max _{k} \delta_{k}+\lambda_{\max }\left(\sum_{v=u-1}^{d} A_{u v} A_{u v}^{T}+(u-1) Q_{k_{u}}\right)}{\lambda_{\max }\left(A_{u u}-\Xi_{u}\right)},
$$

then system (2.5) can be cluster synchronized.

Remark 3.5. Corollary 3.4 gives a sufficient condition for achieving synchronization of linearly coupled complex networks under pinning control, which is different from that obtained by Wu et al. [19, Theorem 2].

As pointed out by $\mathrm{Wu}$ et al. [19], real-world complex networks have coupling strengths that are usually many orders of magnitude less than their theoretically assumed values, and minor modifications are, therefore, required on the above pinning scheme to accommodate for relatively small coupling strengths. Thus by using an adaptive feedback control technique, we have the following theorem.

Theorem 3.6. Suppose that $\varepsilon_{1}, \varepsilon_{2}, \ldots, \varepsilon_{d}$ are d positive constants and

$$
A=\left[\begin{array}{cccc}
A_{11} & A_{12} & \cdots & A_{1 d} \\
A_{21} & A_{22} & \cdots & A_{2 d} \\
\vdots & \vdots & \ddots & \vdots \\
A_{d 1} & A_{d 2} & \cdots & A_{d d}
\end{array}\right] \in \mathbf{M}_{2}
$$

Pick the coupling matrix

$$
A(t)=\left[a_{i j}(t)\right]_{m \times m}=\left[\begin{array}{cccc}
c(t) A_{11} & A_{12} & \cdots & A_{1 d} \\
A_{21} & c(t) A_{22} & \cdots & A_{2 d} \\
\vdots & \vdots & \ddots & \vdots \\
A_{d 1} & A_{d 2} & \cdots & c(t) A_{d d}
\end{array}\right]
$$

with the control strengths

$$
\varepsilon_{l}(t)=c(t) \varepsilon_{l}, \quad l=1,2, \ldots, d,
$$


where $c(t)$ is the adaptive coupling strength. Let $\Delta=\operatorname{diag}\left\{\delta_{1}, \delta_{2}, \ldots, \delta_{n}\right\}$ and $P=\operatorname{diag}\left\{p_{1}, p_{2}\right.$, $\left.\ldots, p_{n}\right\}$ be positive definite diagonal matrices such that $f(x, t) \in Q U A D(P, \Delta) ; h(\cdot) \in U N I(\underline{h}, \bar{h})$ with $\bar{h} \geq \underline{h}>0$. Then the coupled system

$$
\begin{gathered}
\dot{x}_{i}(t)=f\left(x_{i}(t), t\right)+\sum_{j=1}^{m} a_{i j}(t) \Gamma g\left(x_{j}(t)\right)-\varepsilon_{\tilde{i}}(t) \Gamma\left(g\left(x_{i}(t)\right)-g\left(s_{\tilde{i}}(t)\right)\right), \quad i \in J, \\
\dot{x}_{i}(t)=f\left(x_{i}(t), t\right)+\sum_{j=1}^{m} a_{i j}(t) \Gamma g\left(x_{j}(t)\right), \quad i \notin J, \\
\dot{c}(t)=\alpha \sum_{i=1}^{m} e_{i}(t)^{T} P e_{i}(t)
\end{gathered}
$$

can be realized cluster synchronization for $c(0) \geq 0$ and $\alpha>0$.

Proof. Choose a constant $\alpha>0$, and let $e_{i}(t)=x_{i}(t)-s_{\tilde{i}}(t)$. Define a Lyapunov function by

$$
V(t)=\frac{1}{2} \sum_{i=1}^{m} e_{i}(t)^{T} P e_{i}(t)+\frac{1}{2 \alpha \beta}(c-\beta c(t))^{2},
$$

where $c$ and $\beta$ are constants that will be determined later. Differentiating $V(t)$ above gives

$$
\begin{aligned}
& \frac{d V(t)}{d t}=\sum_{i=1}^{m} e_{i}(t)^{T} P \dot{e}_{i}(t)-\alpha \dot{c}(t)+\beta \dot{c}(t) c(t) \\
& =\sum_{i=1}^{m} e_{i}(t)^{T} P\left(f\left(x_{i}(t), t\right)-f\left(s_{\tilde{i}}(t), t\right)+\sum_{j=1}^{m} c(t) a_{i j} \Gamma g\left(x_{j}(t)\right)\right) \\
& -(c-\beta c(t)) \sum_{i=1}^{m} e_{i}(t)^{T} P e_{i}(t)-\sum_{i \in J} c(t) \varepsilon_{\tilde{i}} e_{i}(t)^{T} P \Gamma\left(g\left(x_{i}(t)\right)-g\left(s_{i}(t)\right)\right) \\
& =\sum_{i=1}^{m} e_{i}(t)^{T} P\left(f\left(x_{i}(t), t\right)-f\left(s_{\tilde{i}}(t), t\right)-\Delta e_{i}(t)\right) \\
& +\sum_{i=1}^{m} e_{i}(t)^{T} P \Delta e_{i}(t)+\sum_{i=1}^{m} e_{i}(t)^{T} P \sum_{j=1}^{m} a_{i j}(t) \Gamma g\left(x_{j}(t)\right) \\
& -\sum_{i \in J} c(t) \varepsilon_{i} e_{i}(t)^{T} P \Gamma\left(g\left(x_{i}(t)\right)-g\left(s_{\tilde{i}}(t)\right)\right)-(c-\beta c(t)) \sum_{i=1}^{m} e_{i}(t)^{T} P e_{i}(t),
\end{aligned}
$$


and as in Theorem 3.1, we have

$$
\begin{aligned}
& \sum_{i=1}^{m} e_{i}(t)^{T} P \sum_{j=1}^{m} a_{i j}(t) \Gamma g\left(x_{j}(t)\right) \\
& \quad \leq \sum_{k=1}^{n} p_{k} \gamma_{k} \sum_{u=1}^{d}\left\{\underline{h} c(t) \tilde{e}_{u}^{k}(t)^{T} A_{u u} \tilde{e}_{u}^{k}(t)+\sum_{v>u} \tilde{e}_{u}^{k}(t)^{T} A_{u v} A_{u v}^{T} \tilde{e}_{u}^{k}(t)+\sum_{v<u} \bar{h}^{2} \tilde{e}_{u}^{k}(t)^{T} Q_{k_{u}} \tilde{e}_{u}^{k}(t)\right\} .
\end{aligned}
$$

Inequalities (3.27) and (3.28) imply that

$$
\begin{aligned}
& \frac{d V(t)}{d t} \leq-\eta \sum_{i=1}^{m} e_{i}(t)^{T} e_{i}(t)+\sum_{k=1}^{n} p_{k} \delta_{k} \sum_{u=1}^{d} \tilde{e}_{u}^{k}(t)^{T} \widetilde{e}_{u}^{k}(t) \\
& +\sum_{k=1}^{n} p_{k} \gamma_{k} \sum_{u=1}^{d}\left\{\underline{h} c(t) \tilde{e}_{u}^{k}(t)^{T} A_{u u} \tilde{e}_{u}^{k}(t)\right. \\
& \left.+\sum_{v>u} \tilde{e}_{u}^{k}(t)^{T} A_{u v} A_{u v}^{T} \tilde{e}_{u}^{k}(t)+\sum_{v<u} \bar{h}^{2} \tilde{e}_{u}^{k}(t)^{T} Q_{k_{u}} \tilde{e}_{u}^{k}(t)\right\} \\
& -c(t) \sum_{k=1}^{n} p_{k} \gamma_{k} \sum_{u=1}^{d} \tilde{e}_{u}^{k}(t)^{T} \Xi_{u} \tilde{e}_{u}^{k}(t)-(c-\beta c(t)) \sum_{k=1}^{n} p_{k} \sum_{u=1}^{d} \widetilde{e}_{u}^{k}(t)^{T} \tilde{e}_{u}^{k}(t) \\
& =-\eta \sum_{i=1}^{m} e_{i}(t)^{T} e_{i}(t)+\sum_{k=1}^{n} p_{k} \sum_{u=1}^{d} \tilde{e}_{u}^{k}(t)^{T}\left\{\delta_{k} I_{k_{u}}+\sum_{v=u-1}^{d} \gamma_{k} A_{u v} A_{u v}^{T}+(u-1) \bar{h}^{2} \gamma_{k} Q_{k_{u}}-c I_{u}\right. \\
& \left.+c(t)\left(\underline{h} \gamma_{k} A_{u u}-\underline{h} \gamma_{k} \Xi_{u}+\beta I_{u}\right)\right\} \tilde{e}_{u}^{k}(t) .
\end{aligned}
$$

Thus by choosing $\beta$ and $c$ such that $\underline{h} \gamma_{k} A_{u u}-\underline{h} \gamma_{k} \Xi_{u}+\beta I_{u} \leq 0$ and $\delta_{k} I_{k_{u}}+\sum_{v=u-1}^{d} \gamma_{k} A_{u v} A_{u v}^{T}+$ $(u-1) \bar{h}^{2} \gamma_{k} Q_{k_{u}}-c I_{u} \leq 0$ for any $k=1,2, \ldots, n, u=1,2, \ldots, d$, we obtain

$$
\frac{d V(t)}{d t} \leq-\eta \sum_{i=1}^{m} e_{i}(t)^{T} e_{i}(t)<0
$$

Hence $x_{i}(t) \rightarrow s_{\tilde{i}}(t)$ and $\dot{c}(t) \rightarrow 0$ (by Cauchy's convergence principle), and $c(t)$ converges to some final coupling strength $c_{0}$.

\section{Numerical Simulation}

In this section, we illustrate the theorems of the previous section by numerically simulating some selected cluster complex networks. 


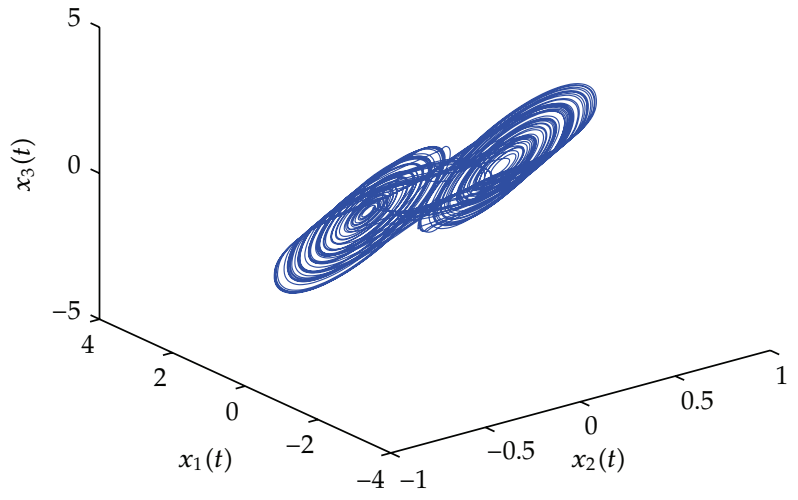

Figure 1: Chua's circuit with $x_{i}(0)=0.1$, for $i=1,2,3$.

\subsection{Cluster Synchronization of Chua'S Circuit by Pinning Control}

The Chua's circuit has been built and used in many laboratories as a physical source of pseudorandom signals, and in numerous experiments on synchronization studies, such as secure communication systems and simulations of brain dynamics. It has also been used extensively in many numerical simulations and exploited in avant-garde music compositions [28] and in the evolution of natural languages [29]. Arrays of Chua's circuits have been used to generate 2-dimensional spiral waves and 3-dimensional scroll waves [30].

The isolated Chua's circuit is described by

$$
\begin{gathered}
\dot{x}_{1}=p\left(x_{2}-x_{1}-g\left(x_{1}\right)\right), \\
\dot{x}_{2}=x_{1}-x_{2}+x_{3}, \\
\dot{x}_{3}=-q x_{2},
\end{gathered}
$$

where $g(x)=m_{0} x+\left(\left(m_{1}-m_{0}\right) / 2\right)(|x+1|-|x-1|)$. We take that $p=10, q=14.87, m_{0}=-0.68$, $m_{1}=-1.27$, and Chua's circuit is chaotic (Figure 1).

Consider a network with 3 clusters and 15 nodes $\left(G_{1}=\{1, \ldots, 5\}, G_{2}=\{6, \ldots, 10\}\right.$, $\left.G_{3}=\{11, \ldots, 15\}\right)$ with the topology structure (Figure 2).

Let $\Xi_{1}=\Xi_{2}=\Xi_{3}=\operatorname{diag}\{10,10,0,0,0,0\}, c=15$, and $s_{1}(t), s_{2}(t)$, and $s_{3}(t)$ be the solutions of the noncoupled system $\dot{s}(t)=f(s(t), t)$ with initial values $s_{1}(0)=[0.1,0.1,0.1]^{T}$, $s_{2}(0)=[0.2,0.2,0.2]^{T}$, and $s_{3}(0)=[0.3,0.3,0.3]^{T}$. The following equality is used to investigate the process of cluster:

$$
E(t)=\frac{1}{18} \sum_{i=1}^{18}\left\|x_{i}(t)-s_{i}(t)\right\|^{2}
$$

If $\lim _{t \rightarrow \infty} E(t)=0$, then we say that the complex network achieves cluster synchronization. The state behavior of the clusters is shown in Figures 3(a), 3(b), 3(c) and the evolution of $E(t)$ in pinning Chua's circuit networks is shown in Figure 3(d). By using adaptive control 


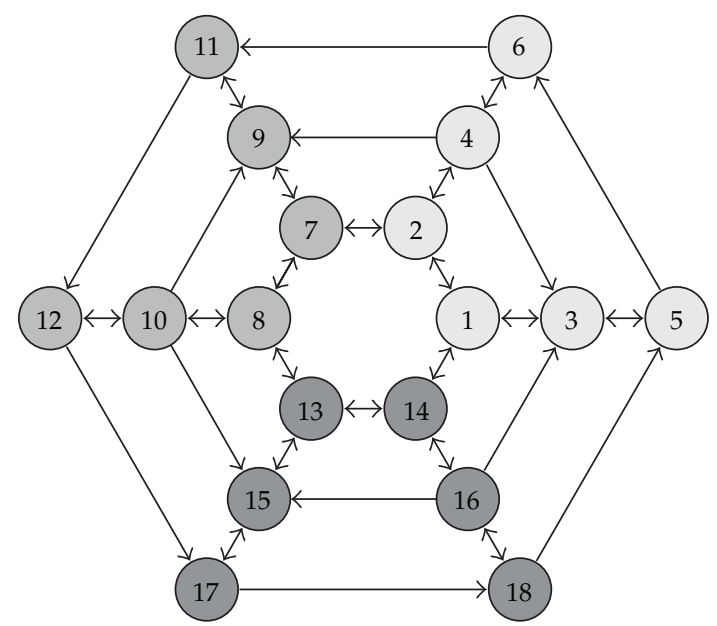

Figure 2: A community network with size $N=18$. There are three communities, each of them is denoted by the dots with gray degree.

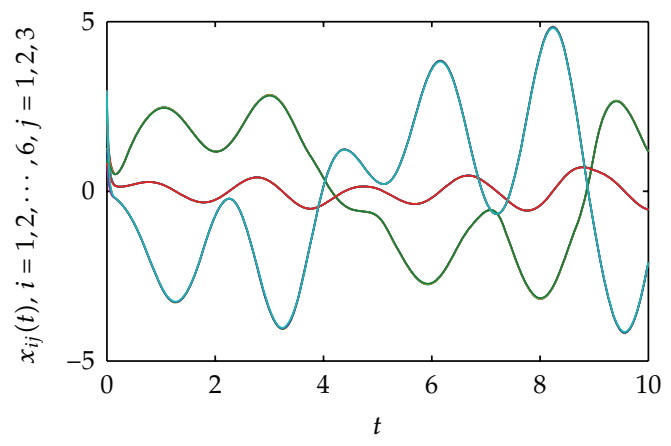

(a)

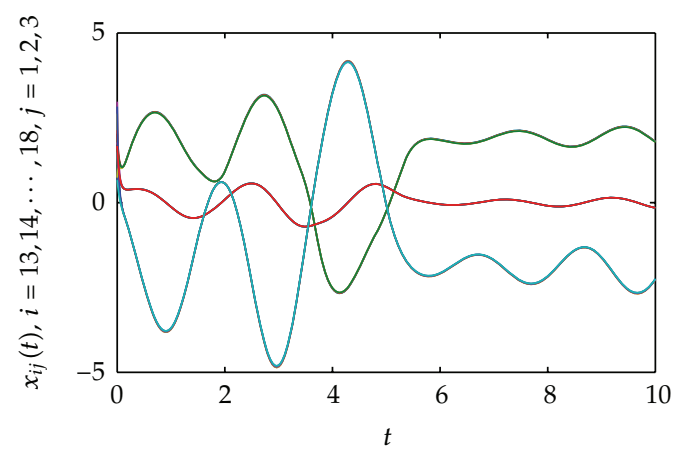

(c)

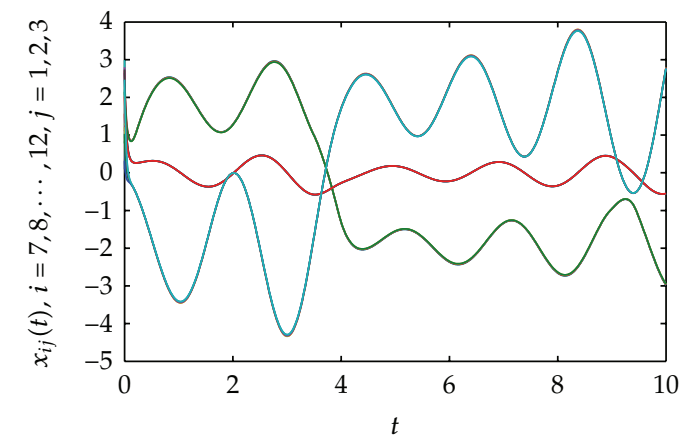

(b)

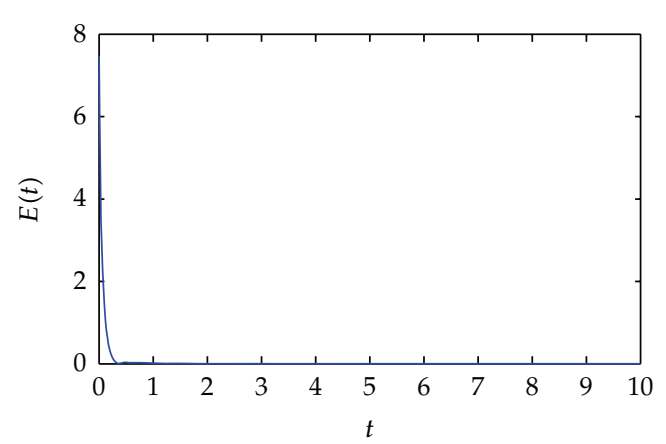

(d)

Figure 3: Cluster synchronization of a nonlinearly coupled Chua's circuit network. $(a, b, c)$ The trajectory of the nodes. (d) The error dynamics of the above Chua's circuit network. 


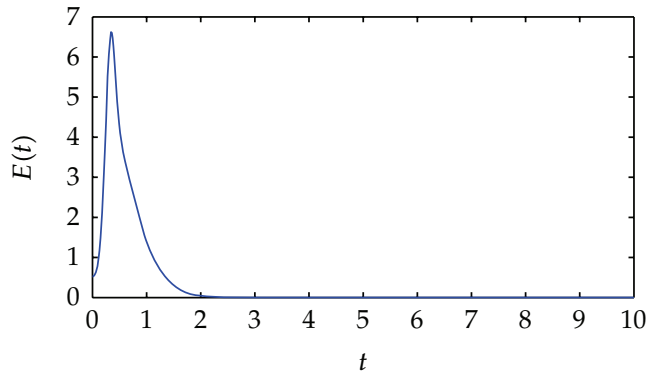

(a)

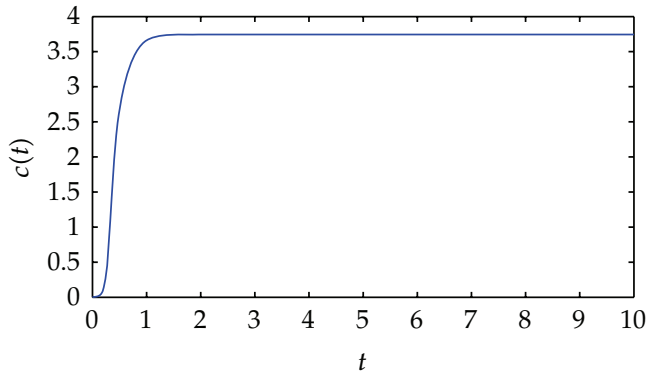

(b)

Figure 4: Adaptive adjustment of the coupling strength of a pinned Chua's circuit network.

scheme, it can be seen that the coupling strength decreases to 3.6 while the original coupling strength is 15 in Figure 4.

\subsection{Cluster Synchronization of Hopfield Neural Network by Pinning Control}

The Hopfield neural network is a simple artificial network which is able to store certain memories or patterns in a manner rather similar to the brain-the full pattern can be recovered if the network is presented with only partial information.

Consider a Hopfield neural network

$$
\frac{d x}{d t}=-D x+T p(x)+I
$$

where $x=\left[x_{1}, x_{2}, x_{3}\right]^{T} \in R^{3}, D=I_{3}$,

$$
T=\left[\begin{array}{ccc}
1.25 & -3.2 & -3.2 \\
-3.2 & 1.1 & -4.4 \\
-3.2 & 4.4 & 1
\end{array}\right]
$$

And $p(x)=\left[q\left(x_{1}\right), q\left(x_{2}\right), q\left(x_{3}\right)\right]^{T}$, where $q(s)=(|s+1|+|s-1|) / 2$ and $I=0$. As in [31], $f(x, t) \in \operatorname{QUAD}\left(5.5682 I_{3}, I_{3}\right)$, and we take the nonlinear coupling $g(x)=5 x+\sin (x)$ and inner coupling matrix $\Gamma=I_{3}$.

Consider a network with 2 clusters and 5 nodes $\left(G_{1}=\{1,2\}, G_{2}=\{3,4,5\}\right)$ with coupling matrix $A \in \mathbf{M}_{2}$, where

$$
A=\left[\begin{array}{ccccc}
-5 & 5 & 1 & 0 & -1 \\
5 & -5 & -1 & 0 & 1 \\
1 & -1 & -10 & 5 & 5 \\
0 & 0 & 5 & -10 & 5 \\
-1 & 1 & 5 & 5 & -10
\end{array}\right] .
$$




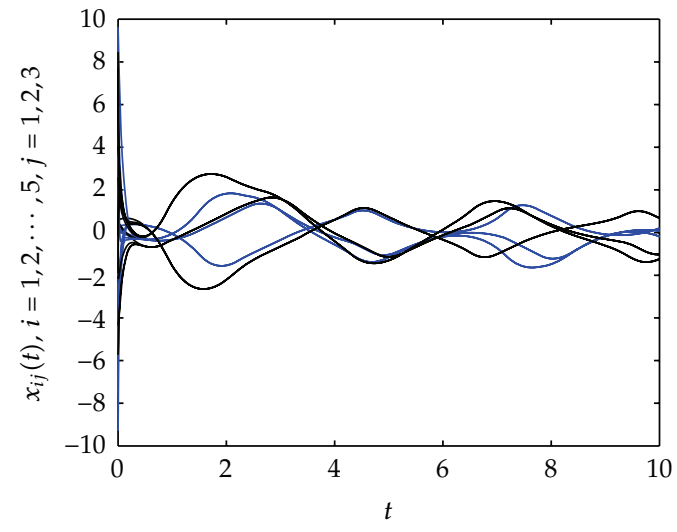

(a)

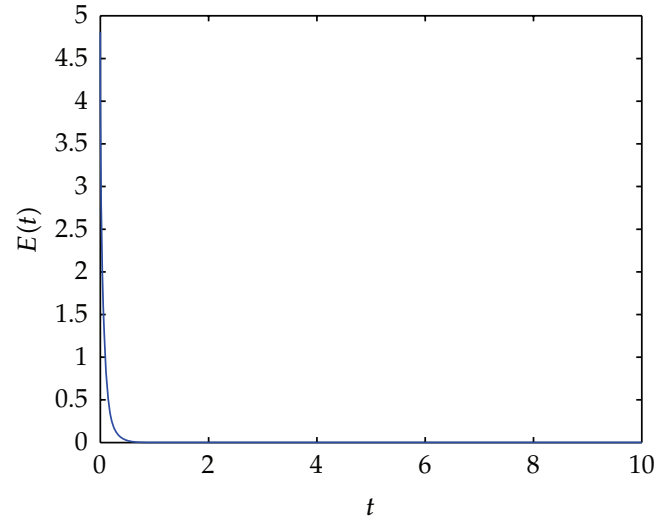

(b)

Figure 5: Cluster synchronization of a nonlinearly coupled Hopfield neural network. (a) The states of the nodes. (b) The error dynamics of the above Hopfield neural network.

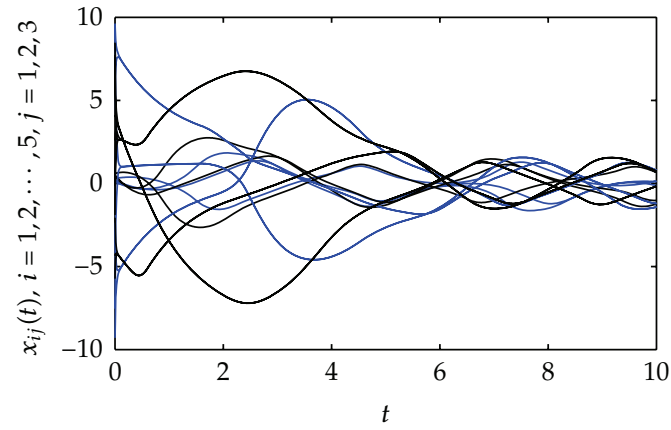

(a)

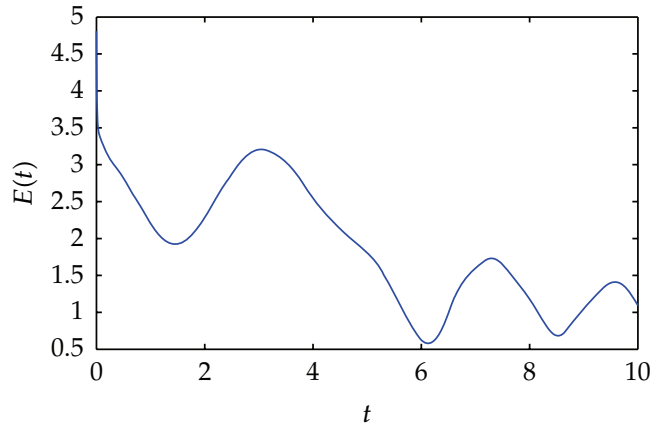

(b)

Figure 6: The trajectory of a nonlinearly coupled Hopfield neural network without pinning control. (a) The states of the nodes. (b) The error dynamics of the above Hopfield neural network.

Let $\Xi_{1}=\operatorname{diag}\{9,0\}$ and $\Xi_{2}=\operatorname{diag}\{9,0,0\}$. Then, condition (3.1) of Theorem 3.1 is satisfied. Let $s_{1}(t)$ and $s_{2}(t)$ be the solutions of the noncoupled system $\dot{s}(t)=f(s(t), t)$ with initial values $s_{1}(0)=[0.1,0.2,0.3]^{T}$ and $s_{2}(0)=[0.4,0.5,0.6]^{T}$. The following equality is used to investigate the process of cluster:

$$
E(t)=\frac{1}{5} \sum_{i=1}^{5}\left\|x_{i}(t)-s_{\tilde{i}}(t)\right\|^{2}
$$

If $\lim _{t \rightarrow \infty} E(t)=0$, then we say that the complex network achieves cluster synchronization. The state behavior of the first and second clusters is shown, respectively, by the blue and black curves in Figure 5(a), and the evolution of $E(t)$ in pinning Hopfield neural networks is shown in Figure 5(b). The state behavior of the Hopfield neural networks is shown in Figure 6(a), and the evolution of $E(t)$ in pinning Hopfield neural networks is shown in Figure 6(b). The 


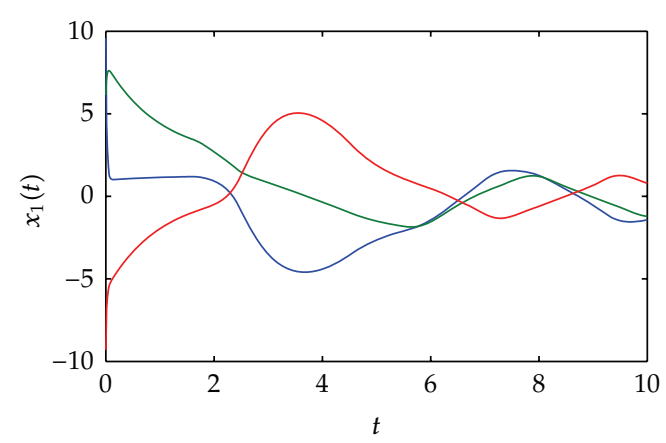

(a)

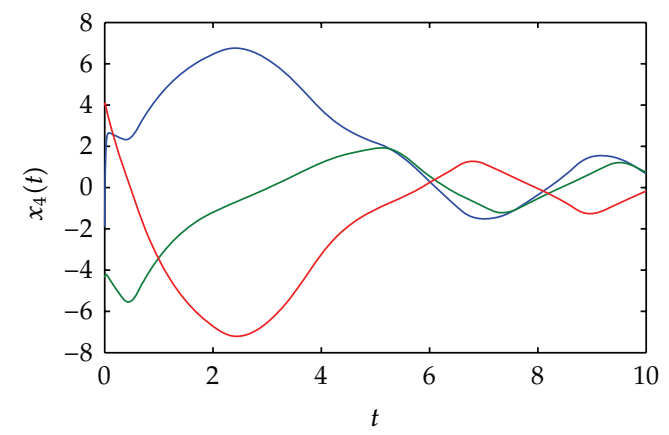

(c)

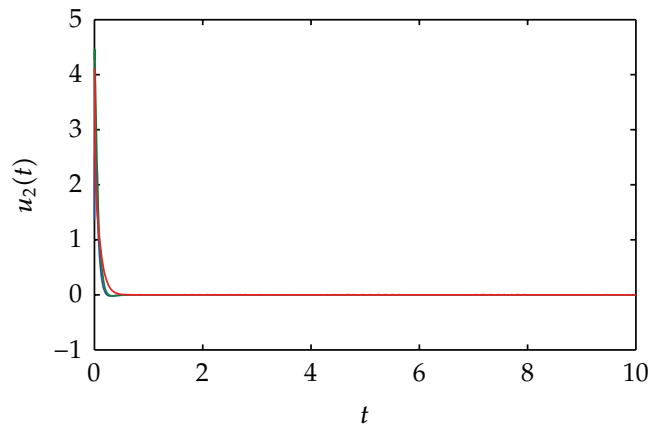

(b)

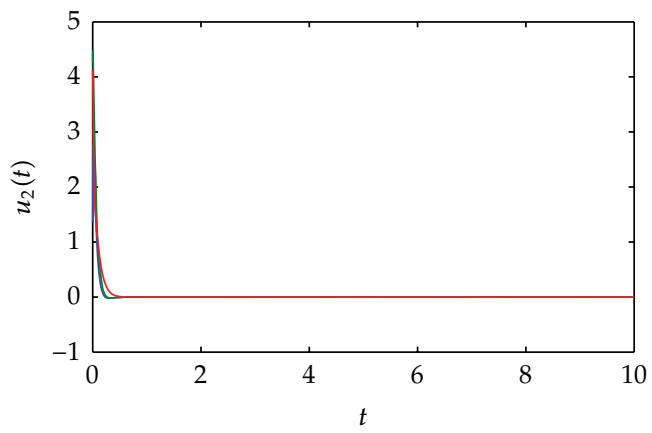

(d)

Figure 7: (a) The trajectory of $x_{1}$ in a nonlinearly coupled Hopfield neural network without pinning control. (b) The signal added on $x_{1}$ in the above Hopfield neural network. (c) The trajectory of $x_{4}$ in a nonlinearly coupled Hopfield neural network without pinning control. (d) The signal added on $x_{4}$ in the above Hopfield neural network.

signal is compared with the trajectory of a nonlinearly coupled Hopfield neural network without pinning control in Figure 7.

Then, we discuss the cluster synchronization of a Hopfield neural network by adaptive pinning control. In this simulation, the initial values of the network nodes are randomly drawn (and then fixed) from the standard uniform distribution on the open interval $(0,1)$, and $s_{1}(t), s_{2}(t)$, and $s_{3}(t)$ are the solutions of the uncoupled system $\dot{s}(t)=f(s(t), t)$ with initial values $s_{1}(0)=[0.1,0.2,0.3]^{T}, s_{2}(0)=[0.4,0.5,0.6]^{T}$, and $s_{3}(0)=[0.7,0.8,0.9]^{T}$. In the topological structure of the network (Figure 10(a)), we select $l_{1}=9, \varepsilon_{1}=37, l_{2}=8, \varepsilon_{2}=26$, $l_{3}=9$, and $\varepsilon_{3}=27$; then we can calculate $c_{1}=31.61545, c_{2}=31.7185, c_{3}=31.8915$ from inequalities (3.19) in Corollary 3.3. Thus, the condition for achieving cluster synchronization is satisfied if $c>31.8915$. We choose the coupling strength $c=32$.

Let

$$
E(t)=\frac{1}{300} \sum_{i=1}^{300}\left\|x_{i}(t)-s_{\tilde{i}}(t)\right\|^{2} .
$$

Figure 8 shows the time evolution of $E(t)$ and $c(t)$ for the pinned Hopfield neural network (4.3) with initial values that were randomly chosen from the interval $[0,1]$. The 


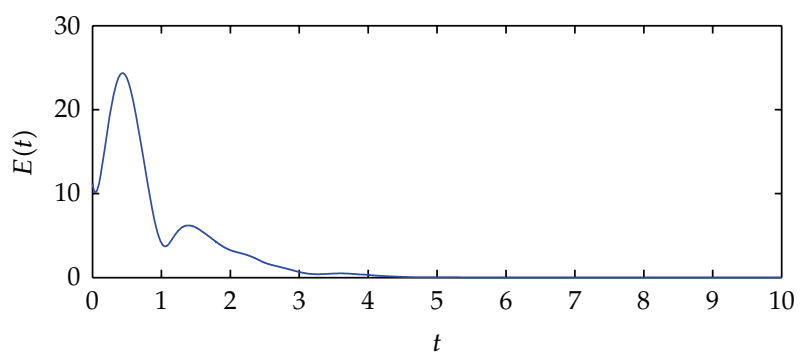

(a)

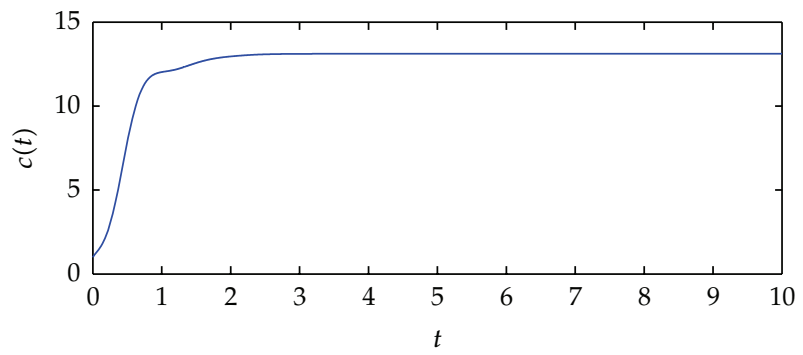

(b)

Figure 8: Adaptive adjustment of the coupling strength of a pinned Hopfield neural network.

coupling strength is designed by using the adaptive technique given in Theorem 3.6, and the final coupling strength is $c=12.8706$.

Next, we present the cluster synchronization for the same systems with different coupling strength by numerical simulation. Figure 9(a) shows $E(t)$ with $c=12.8706$ and Figure 9(b) shows $E(t)$ with $c=32$ for the same initial values as those in Figure 8. Clearly, cluster synchronization is attained much faster for the network with $c=32$ than for the one with $c=12.8706$ in Figures 9(a) and 9(b). One can see that under the same network system as above, if we take $c=5.3602$, the cluster synchronization fails (Figure 9(c)).

Remark 4.1. By using the adaptive technique, we get a final coupling strength, with which the cluster synchronization is realized (Figure 9(a)). Obviously, it is smaller than theoretical coupling strength. In practice, the final coupling strength is very effective, since the synchronization cost is lower than the cluster synchronization with the theoretical coupling strength. If the coupling strength is smaller than it, the cluster synchronization probably fails (Figure 9(c)).

\subsection{The Critical Combination of the Control Strength, the Number of Pinned Nodes, and the Coupling Strength}

In this simulation, we consider a network with 3 clusters and 300 nodes $\left(G_{1}=\{1,2, \ldots, 100\}\right.$, $G_{2}=\{101,102, \ldots, 200\}$, and $\left.G_{3}=\{201,202, \ldots, 300\}\right)$ with a topological structure that is shown in Figure 10(a). Specifically, the network is obtained by integrating three scale-free networks that are held together by common edges the exact number of which is proportional to the degree of the nodes. Using the topological structure of the network (Figure 10(a)), we construct the coupling matrix $A \in \mathbf{M}_{\mathbf{2}}$ such that $a_{i j}>0$ if the point $(i, j)$ in Figure 10(a) is "." 


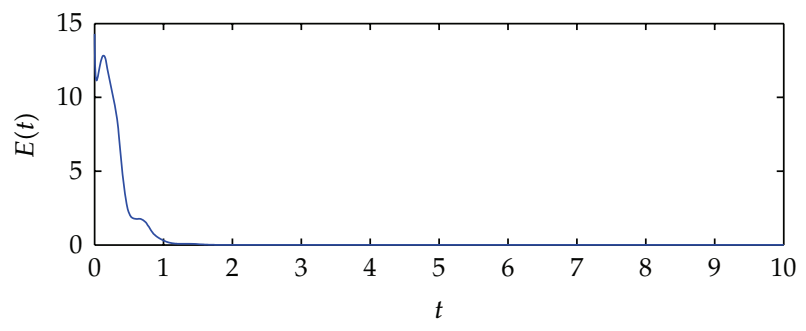

(a) $c=12.8706$

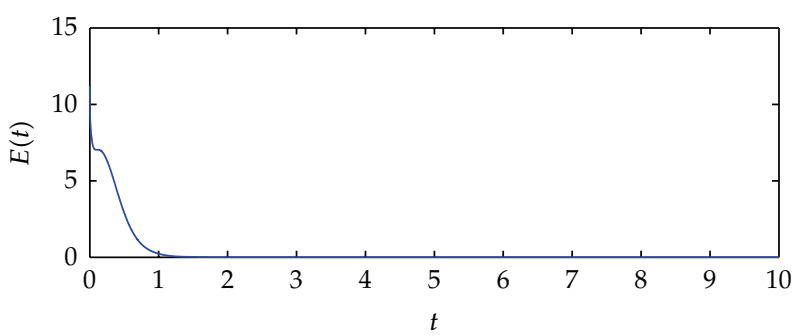

(b) $c=32$

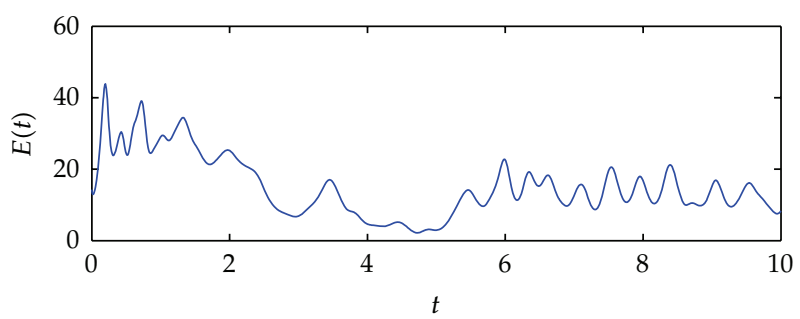

(c) $c=5.3602$

Figure 9: The cluster synchronization error under different coupling strengths.

and $a_{i j}<0$ if the point $(i, j)$ is " $x$ " (Style online). Following [32], no more than one quarter of the cluster nodes are pinned in every cluster in this experiment and we choose to pin those nodes with the largest degrees. Finally, we reject all coupling strengths that are greater than a certain fixed constant (chosen to be 75 in this experiment) because the coupling strengths cannot be too large in practice. A critical value for the coupling strength $c_{u}$ is then calculated (using Corollary 3.3) for every critical combination of the number of pinned nodes $l_{u}$ and the control strength $\varepsilon_{u}$ for every cluster.

We see in Figures 10(b), 10(c), and 10(d) that even though the color RGB(128,0,0) represents the unacceptable coupling strengths 75 , many combinations of the coupling strength, the number of pinned nodes and the control strength can still be taken to realize cluster synchronization. Furthermore, for every cluster if the number of pinned nodes $l_{u}$ is determined, the coupling strength $c_{u}$ decreases as the control gain $\varepsilon_{u}$ increases, and finally it approaches a constant. Once the control strength $\varepsilon_{u}$ is determined, the coupling strength $c_{u}$ decreases as the number of nodes $l_{u}$ increases, and finally it approaches a constant. Furthermore, if the coupling strength $c_{u}$ approaches the threshold, it almost has no changes no matter how you increase the control strength $l_{u}$ and the number of pinned nodes $l_{u}$. In 


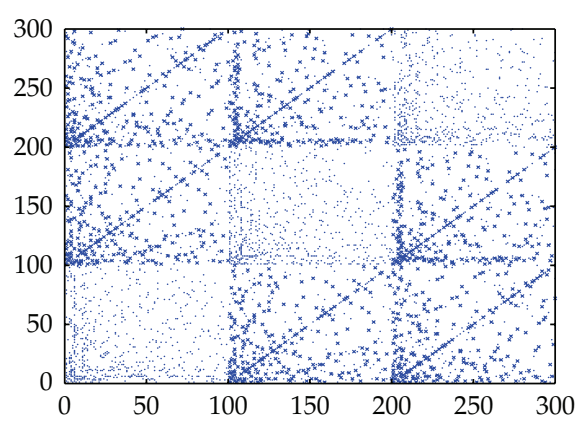

(a) Topological structure of the complex network

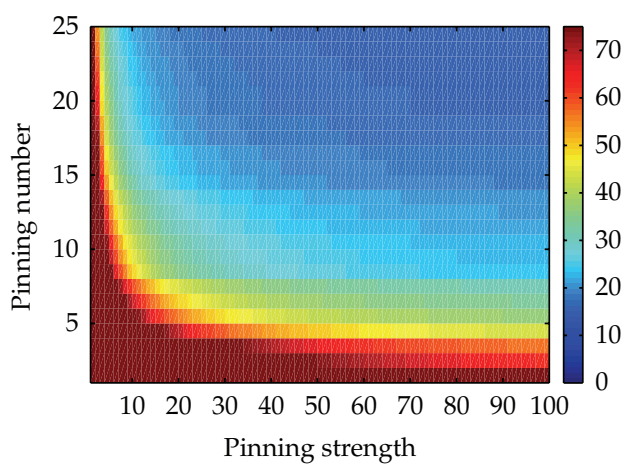

(c) $l_{2}-\varepsilon_{2}-c_{2}$ for $u=2$

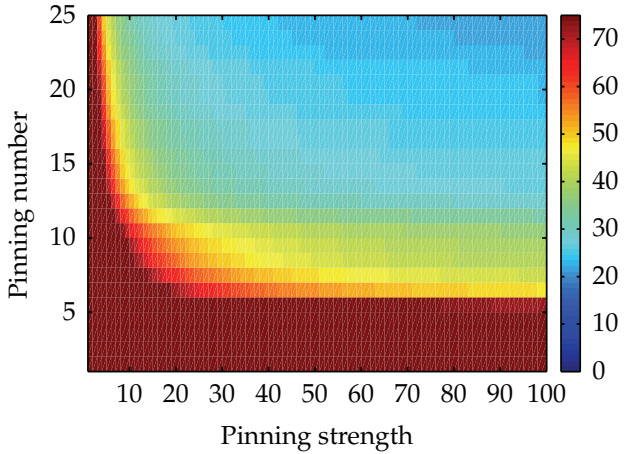

(b) $l_{1}-\varepsilon_{1}-c_{1}$ for $u=1$

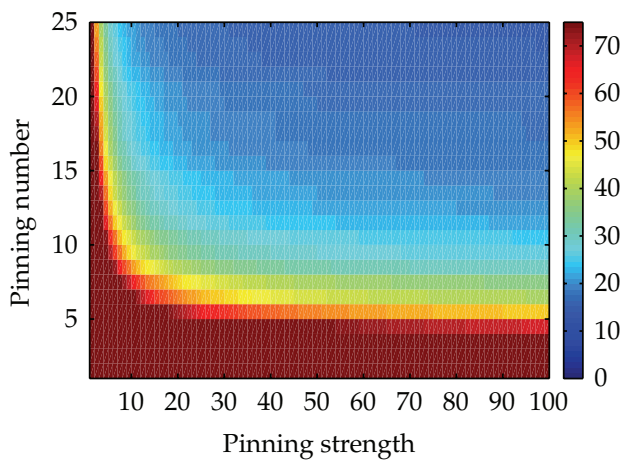

(d) $l_{3}-\varepsilon_{3}-c_{3}$ for $u=3$

Figure 10: (Color and style online) $l_{u}-\varepsilon_{u}-c_{u}$ expresses the critical combination of the coupling strength, the number of pinned nodes, and the control strength of the $u$ th cluster. (b) The relationship between $\varepsilon, l$ and $c$ of the first cluster. (c) The second cluster. (d) The third cluster.

particular, a certain combination of suitable $l_{u}, \varepsilon_{u}$, and $c_{u}$ for every cluster exists that will minimize the synchronization cost. It is a challenging problem to find the minimal cost combination for a specific complex network.

\section{Conclusion}

In this paper, we investigated the cluster synchronization problem for complex networks that are nonlinearly coupled. Specifically, we achieved global cluster synchronization by applying a pinning control scheme to every individual cluster and derived sufficient conditions for the global stability of cluster synchronization. Furthermore, in view of the great disparity in the coupling strength magnitudes that exist between theoretical and real-world systems, we also rigorously proved an adaptive feedback control technique that can be used to completely cluster synchronize any real-world network. Finally, for clarity of exposition, some numerical examples were considered that illustrate the theoretical analysis and the acceptance condition was given for the number of pinned nodes and coupling and control strengths of one particular network. 


\section{Acknowledgments}

This work was supported by the National Science Foundation of China under Grant no. 61070087, Guangdong Education University Industry Cooperation Projects (2009B090300355) and Shenzhen Basic Research Project (JC200903120040A, JC201006010743A), and a research fund form the Hong Kong Polytechnic University. The authors are very grateful to reviewers and the editor for their valuable comments and suggestions to improve the presentation of the paper.

\section{References}

[1] L. Kuhnert, K. I. Agladze, and V. I. Krinsky, "Image processing using light-sensitive chemical waves," Nature, vol. 337, pp. 244-247, 1989.

[2] S. H. Strogatz and I. Stewart, "Coupled oscillators and biological synchronization," Scientific American, vol. 269, no. 6, pp. 102-109, 1993.

[3] C. M. Gray, "Synchronous oscillations in neuronal systems: mechanisms and functions," Journal of Computational Neuroscience, vol. 1, no. 1-2, pp. 11-38, 1994.

[4] M. D. Vieira, "Chaos and synchronized chaos in an earthquake model," Physical Review Letters, vol. 82, no. 1, pp. 201-204, 1999.

[5] L. Glass, "Synchronization and rhythmic processes in physiology," Nature, vol. 410, pp. 277-284, 2001.

[6] S. Wang, J. Kuang, J. Li, Y. Luo, H. Lu, and G. Hu, "Chaos-based secure communications in a large community," Physical Review E, vol. 66, no. 6, Article ID 065202, 4 pages, 2002.

[7] D. Yu, M. Righero, and L. Kocarev, "Estimating topology of networks," Physical Review Letters, vol. 97, no. 18, Article ID 188701, 4 pages, 2006.

[8] S. Boccaletti, J. Kurths, G. Osipov, D. L. Valladares, and C. S. Zhou, "The synchronization of chaotic systems," Physics Reports A, vol. 366, no. 1-2, pp. 1-101, 2002.

[9] Z. Zheng and G. Hu, "Generalized synchronization versus phase synchronization," Physical Review E, vol. 62, pp. 7882-7885, 2000.

[10] M. G. Rosenblum, A. S. Pikovsky, and J. Kurths, "From phase to lag synchronization in coupled chaotic oscillators," Physical Review Letters, vol. 78, no. 22, pp. 4193-4196, 1997.

[11] V. N. Belykh, I. V. Belykh, and E. Mosekilde, "Cluster synchronization modes in an ensemble of coupled chaotic oscillators," Physical Review E, vol. 63, no. 3, part 2, Article ID 036216, 2001.

[12] M. G. Rosenblum, A. S. Pikovsky, and J. Kurths, "Phase synchronization of chaotic oscillators," Physical Review Letters, vol. 76, no. 11, pp. 1804-1807, 1996.

[13] C. Vreeswijk, "Partial synchronization in populations of pulse-coupled oscillators," Physical Review E, vol. 54, no. 5, pp. 5522-5537, 1996.

[14] B. Ao and Z. Zheng, "Partial synchronization on complex networks," Europhysics Letters, vol. 74, no. 2, Article ID 50006, 2006.

[15] L. M. Pecora and T. L. Carroll, "Synchronization in chaotic systems," Physical Review Letters, vol. 64, no. 8, pp. 821-824, 1990.

[16] N. F. Rulkov, M. M. Sushchik, and L. S. Tsimring, "Generalized synchronization of chaos in directionally coupled chaotic systems," Physical Review E, vol. 51, no. 2, pp. 980-994, 1995.

[17] A. E. Hramov and A. A. Koronovskii, "An approach to chaotic synchronization," Chaos, vol. 14, no. 3, pp. 603-610, 2004.

[18] Z. Ma, Z. Liu, and G. Zhang, "A new method to realize cluster synchronization in connected chaotic networks," Chaos, vol. 16, no. 2, Article ID 023103, 9 pages, 2006.

[19] W. Wu, W. Zhou, and T. Chen, "Cluster synchronization of linearly coupled complex networks under pinning control," IEEE Transactions on Circuits and Systems I, vol. 56, no. 4, pp. 829-839, 2009.

[20] W. Lu, B. Liu, and T. Chen, "Cluster synchronization in networks of coupled nonidentical dynamical systems," Chaos, vol. 20, no. 1, Article ID 013120, 12 pages, 2010.

[21] W. Lu, B. Liu, and T. Chen, "Cluster synchronization in networks of distinct groups of maps," European Physical Journal B, vol. 77, no. 2, pp. 257-264, 2010.

[22] Z. G. Zheng, X. Q. Feng, B. Ao, and M. C. Cross, "Synchronization of groups of coupled oscillators with sparse connections," Europhysics Letters, vol. 87, no. 5, Article ID 50006, 2009.

[23] K. Wang, X. Fu, and K. Li, "Cluster synchronization in community networks with nonidentical nodes," Chaos, vol. 19, no. 2, Article ID 023106, 10 pages, 2009. 
[24] X. Liu and T. Chen, "Synchronization analysis for nonlinearly-coupled complex networks with an asymmetrical coupling matrix," Physica A, vol. 387, no. 16-17, pp. 4429-4439, 2008.

[25] T. Chen, X. Liu, and W. Lu, "Pinning complex networks by a single controller," IEEE Transactions on Circuits and Systems I, vol. 54, no. 6, pp. 1317-1326, 2007.

[26] W. Guo, F. Austin, and S. Chen, "Global synchronization of nonlinearly coupled complex networks with non-delayed and delayed coupling," Communications in Nonlinear Science and Numerical Simulation, vol. 15, no. 6, pp. 1631-1639, 2010.

[27] Z. Wang, H. Shu, Y. Liu, D. W. C. Ho, and X. Liu, "Robust stability analysis of generalized neural networks with discrete and distributed time delays," Chaos, Solitons \& Fractals, vol. 30, no. 4, pp. 886896, 2006.

[28] E. Bilotta, S. Gervasi, and P. Pantano, "Reading complexity in Chua's oscillator through music. I. A new way of understanding chaos," International Journal of Bifurcation and Chaos, vol. 15, no. 2, pp. 253-382, 2005.

[29] E. Bilotta and P. Pantano, "The language of chaos," International Journal of Bifurcation and Chaos, vol. 16, no. 3, pp. 523-557, 2006.

[30] R. N. Madan, Chuas Circuit: a Paradigm for Chaos, World Scientific, Singapore, 1993.

[31] W. Wu and T. Chen, "Global synchronization criteria of linearly coupled neural network systems with time-varying coupling," IEEE Transactions on Neural Networks, vol. 19, no. 2, pp. 319-332, 2008.

[32] J. Zhao, J. Lu, and X. Wu, "Pinning control of general complex dynamical networks with optimization," Science China Information Sciences, vol. 53, no. 4, pp. 813-822, 2010. 


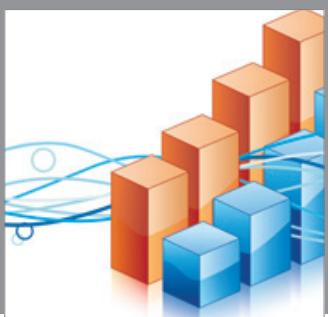

Advances in

Operations Research

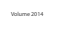

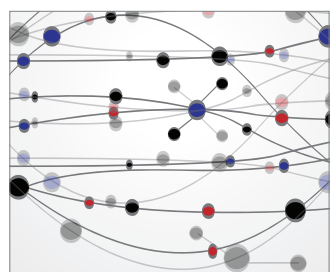

\section{The Scientific} World Journal
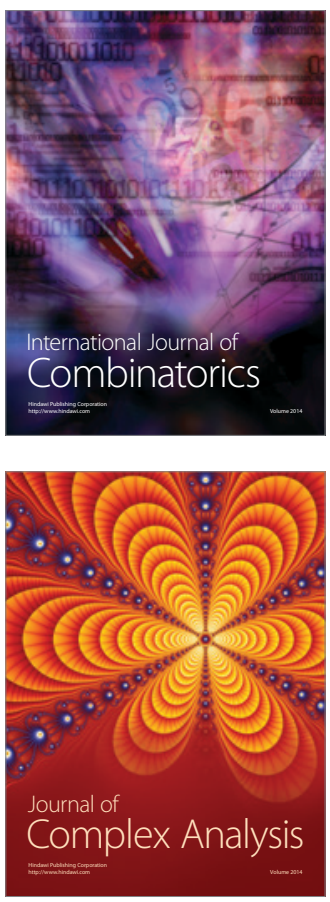

International Journal of

Mathematics and

Mathematical

Sciences
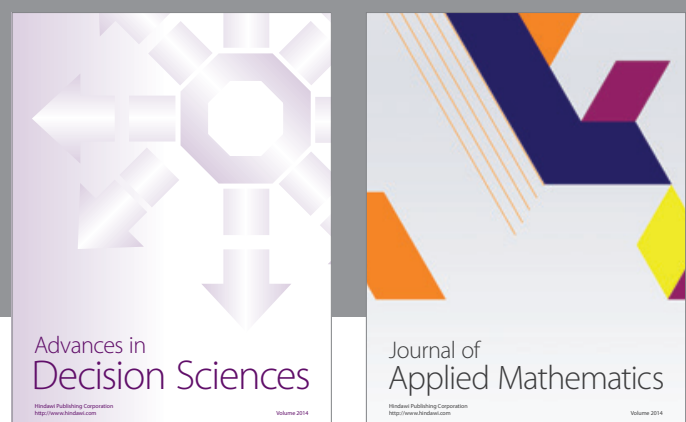

Journal of

Applied Mathematics
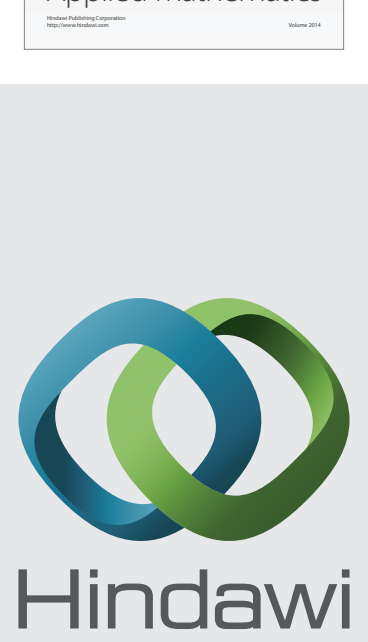

Submit your manuscripts at http://www.hindawi.com
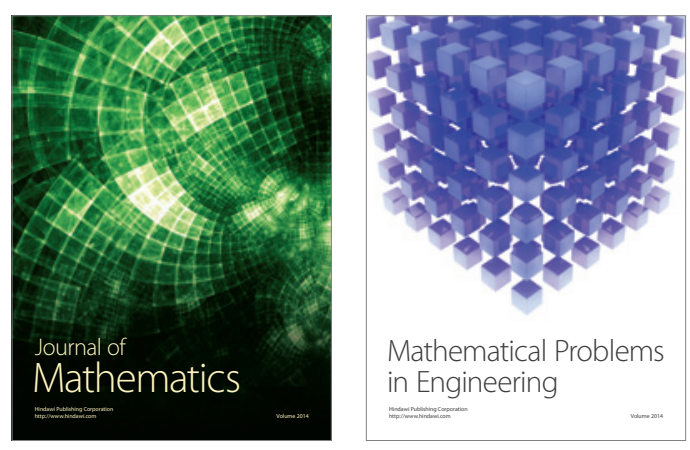

Mathematical Problems in Engineering
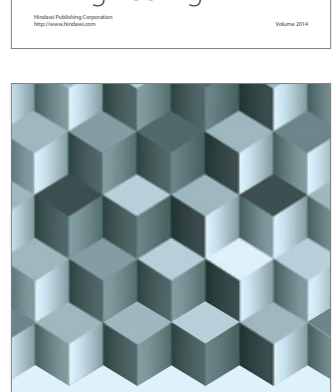

Journal of

Function Spaces
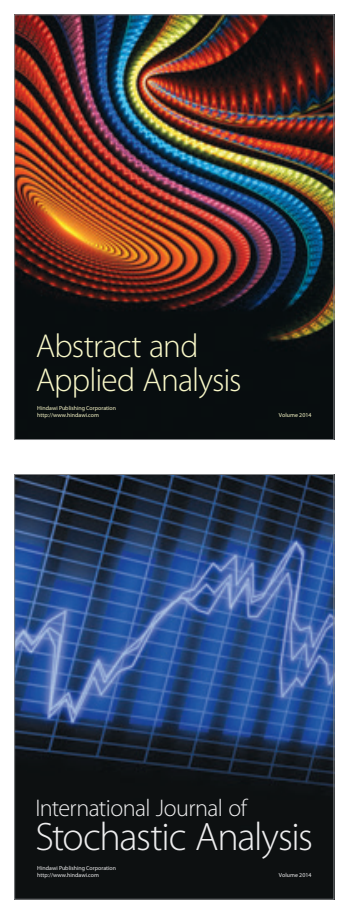

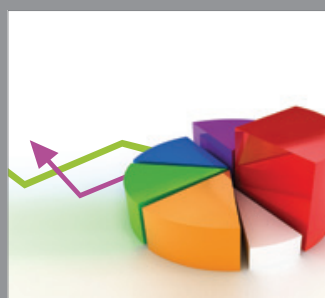

ournal of

Probability and Statistics

Promensencen
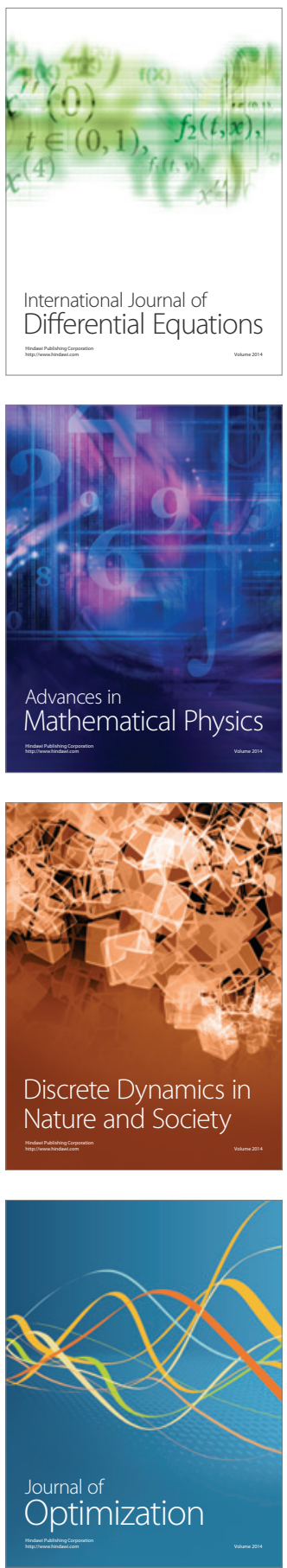